\title{
The Status of Questions in the Ontology of Scientific Change
}

\author{
William Rawleigh \\ IHPST, University of Toronto \\ will.rawleigh@mail.utoronto.ca
}

\section{Keywords}

theoretical scientonomy, ontology of epistemic elements, question, status of questions, theory, proposition, semantics, question acceptance

\begin{abstract}
The currently accepted scientonomic ontology includes two classes of epistemic elements - theories and methods. However, the ontology underlying the Encyclopedia of Scientonomy includes questions/topics as a basic element of its semantic structure. Ideally there should be no discrepancy between the accepted ontology of theoretical scientonomy and that of the Encyclopedia. I argue that questions constitute a distinct class of epistemic elements as they are not reducible to other elements that undergo scientific change - theories or methods. I discuss and reject two attempts at reducing questions to either descriptive or normative theories. According to the descriptive-epistemic account, scientific questions can be logically reduced to descriptive propositions, while according to the normative-epistemic account, they can be reduced to normative propositions. I show that these interpretations are incapable of capturing the propositional content expressed by questions; any possible reduction is carried at the expense of losing the essential characteristic of questions. Further, I find that the attempts to reduce questions to theories introduce an infinite regress, where a theory is an attempt to answer a question, which is itself a theory which answers another question, ad infintum. Instead, I propose to incorporate the question-answer semantic structure from erotetic logic in which questions constitute a distinct class of elements irreducible to propositions. An acceptance of questions into scientonomic ontology as a separate class of epistemic elements suggests a new avenue of research into the mechanism of question acceptance and rejection, i.e. how epistemic communities come to accept certain questions as legitimate and others as illegitimate.
\end{abstract}




\section{Introduction}

At the time of writing there is a problem in particle physics called the strong CP problem that can be roughly formulated in non-technical terms as 'why are there no observed instances of CP-violation (charge parity violation) in quantum chromodynamics (QCD), even though nothing about QCD prohibits it?' (Kim and Carosi, 2010). CP-symmetry states that in any quantum system, if a particle is swapped for its antiparticle - that is to say, if the charge of the particle is reversed - the laws of physics should be the same as long as the spatial co-ordinates of the particle are also reversed. The reversal of the charge between the particle and antiparticle is an instance of $\mathrm{C}$-symmetry, while the reversal of the spatial coordinates is an instance of P-symmetry. In some interactions involving the weak nuclear force the symmetry is broken, leading to an imbalance between matter and antimatter in the system. The Strong CP problem arises when we start looking at CP-violation in QCD, the set of theories that relate to the strong nuclear force. Nothing in these theories prohibits CP-symmetry violations, yet they are never observed in nature. Recent research has pursued a solution based on axions, a hypothetical particle that, if existent, would balance the equations in QCD that suggest CP-violation should take place, thereby also solving problems associated with dark matter and early universe cosmology (Kim \& Carosi, 2010). At this point, however, it is an open question as to what the solution to the problem is, if one even exists. Nonetheless, the physics community accepts that the problem is legitimate and significant amounts of research time and resources have been devoted to finding a solution.

Outlining the potential solutions to the problem as well as discussing which one of them is correct is well outside the scope of this paper. Yet the mere fact that the physics community accepts the question as legitimate illuminates a significant issue for theoretical scientonomy as it presently stands: how do questions like the strong CP problem fit into the ontology of epistemic elements accepted in theoretical scientonomy? Given that our presently accepted ontology contains only theories and methods, we must either reduce questions to theories or methods, or accept questions as distinct epistemic elements of our scientonomic ontology.

The question of questions, so to speak, impacts both the correspondence of our theory to actual scientific practice and the practical implementation of our knowledge viz-a-viz the Encyclopedia of Scientonomy, which has a topic-theory semantics built into its operation. In the encyclopedia every theory is framed as being an answer to some question/topic. Consider the encyclopedia entry on Sebastien's formulation of the third law. The page, along with every other theory page, refers explicitly to the question to which the theory is an answer. In this case, Sebastien's formulation of the third law is an attempt to answer the question on the mechanism of method employment. This presents us, theoretical scientonomists, with a challenge. Ideally, the ontology that is in the backbone of the Encyclopedia of Scientonomy should be in agreement with the official ontology of scientific change accepted in theoretical scientonomy. Nevertheless, we find that the actual ontology of the encyclopedia is not that of theories and methods but includes questions/topics as a basic element of its semantic structure. How are we to reconcile this fact with the reflexivity inherently demanded by scientonomic theory and practice?

In this paper, I will show that the present scientonomic ontology of epistemic elements is ill-equipped to accommodate questions due to the overwhelming problems that occur when attempting to divorce the semantic content of a question from its interrogative nature and its confusion with the question's historical record. Further, I will show that questions are not reducible to theories and that attempts to do so result in an infinite regress. Consequently, I will propose that questions be accepted into the scientonomic ontology as a distinct class of epistemic elements alongside theories and methods.

\section{Background}

In the Encyclopedia of Scientonomy, each topic is characterized by a specific question; questions are precise, interrogative formulations of topics. Throughout this essay, I will use the terms question and topic synonymously since each topic can essentially be formulated as a question. Thus, I tentatively suggest that we define question as "a topic of inquiry": 


\section{Question $\equiv$}

A topic of inquiry.

This definition, albeit extremely broad, reflects how questions/topics are treated in the encyclopedia.

Questions as separate epistemic entities have rarely been a focus of the traditional philosophy of science discourse. Among those few authors who discussed the issue of questions in the philosophy of science were Kuhn and Hempel - in the contexts of scientific change and explanation, respectively. Hempel famously posited explanations as answers to why-questions (Hempel, 1989) but regarded the matter of questions themselves as basic. For Kuhn, any time a paradigm shift occurs in a scientific discipline the sorts of questions (puzzles) that one can ask (attempt to solve) shift with it; in other words, the subjects of normal science shift with the paradigm, but this is as far as Kuhn investigated. As such, the issue of whether or not questions themselves can be

In The Structure of Scientific Revolutions Kuhn writes: "That scientists do not usually ask or debate what makes a particular problem or solution legitimate tempts us to suppose that, at least intuitively, they know the answer. But it may only indicate that neither the question nor the answer is felt to be relevant to their research" (Kuhn, 1970, p. 46). Kuhn, unlike many of his contemporaries, did not disregard the issue of questions entirely. That said, he did not undertake to investigate questions on their own or describe their behavior except as being derived from theories. accommodated into the ontology of scientific change as such has gone largely unexplored, even by those who make the study of scientific change their primary area of research. In our currently accepted theoretical scientonomy, the core elements of science are regarded as being theories and methods (Sebastian, 2016). However, the case of the strong $\mathrm{CP}$ problem illustrates that this ontology may be insufficient when it comes to handling what scientists regard as being a legitimate question or a legitimate research topic.

It is worth reiterating that in the case of the strong CP problem there is a clear consensus among physicists that the problem is legitimate and worthy of study. There is research into the problem going on as you read this, and physicists are openly engaged in discussion of the problem in journal articles and conference proceedings. In other words, the indicators of acceptance we normally associate with theories (Barseghyan, 2015, pp. 113-120) apply equally well to the questions. These indicators reveal that the community of particle physicists accepts the legitimacy of the strong CP problem, that the community will accept, use, or pursue attempts to solve the problem, and that the community will apply the employed method of the time in evaluating whether any solution to the problem is acceptable. If these indicators are to be taken seriously - and I see no reason why they should not be - then we are left with a picture of science where communities are in a position to accept questions in the same way they accept theories. In scientonomy, we accept unambiguously that theories are elements of a mosaic, but currently we do not admit questions to be elements of the mosaic, despite their sharing many common features.

At present, the accepted definition for theory is "a set of propositions" (Sebastien, 2016) and the accepted definition an employed method is roughly the "requirements [that] constitute the actual expectations of the community" (Patton, Overgaard, \& Barseghyan, 2017). These are taken to be the basic epistemic elements of the scientonomic ontology, so there are four avenues that we may take when dealing with the problem of the ontological status of questions:

- Reject that questions can be part of a mosaic.

- Accommodate questions under the umbrella of methods.

- Accommodate questions under the umbrella of theories.

- Accept questions into the scientonomic ontology as a distinct class of epistemic elements.

Let us consider each of these avenues in turn.

The first avenue is unattractive for several reasons. For one, it contradicts the actual practice of science. As we have already discussed, it seems that questions are an integral part of the process of scientific change; otherwise the question "why are there no observed instances of CP violation in QCD in QCD?" would bear no relation to the sorts of theories that physicists in QCD research are pursuing. Any historical reconstruction of the current mosaics of the physics community will be incomplete without mentioning this question. Secondly, it contradicts the semantic structure of the encyclopedia, and therefore contradicts our actual scientonomic practice. 
Topics are built into the semantic structure of the encyclopedia, but that structure does not correspond to the ontology that is actually deployed in our practice of both theoretical and observational scientonomy.

The history of science is replete with examples of questions that have been considered legitimate in some time-periods and illegitimate in others. For instance, in the $18^{\text {th }}$ century before the work of Priestly it would have been perfectly legitimate for a pneumatic chemist to ask "what is the weight of phlogiston?" or "why does some matter gain mass as it loses phlogiston?". The chemists of the time could legitimately spend years of laborious research on trying to answer these questions. However, after the acceptance of the oxidation theory of combustion and the rejection of the phlogiston theory, these questions lost their meaning and became illegitimate. Similarly, an Aristotelian astronomer in the Renaissance could have reasonably contemplated the question "what is the distance from the earth to the sphere of stars?" and done calculations to find an answer. Today, however, we would never consider this question legitimate as we no longer accept a cosmological theory that posits the existence of the sphere of stars. Likewise, the question "what's the underlying mechanics of the evolution of species?" is considered nowadays a legitimate topic of biological research, but would have been deemed meaningless and, thus, illegitimate even some three hundred years ago. In short, the legitimacy of questions changes with time which indicates that they must play an important role in the process of changes in theories and methods. Thus, excluding questions from the scientonomic discourse is simply not an option; we have to find a way to include them into the ontology of epistemic elements.

The second avenue is to try and accommodate questions into the mosaic under the umbrella of methods. This option is similarly unattractive since it requires us to reduce questions to methods. This is a non-starter because of the inherently normative character of methods. Methods, as a rule, have the form of a hypothetical imperative that says accept a theory if $x$, where $x$ is the criteria set out by the employed method. By definition, a question cannot be a conditional/hypothetical imperative as questions list no conditions. A single question, like "what is the spin of an electron?" cannot be stated in terms of a conditional with "accept the theory if..." as the antecedent. This is a simple matter of logical syntax and warrants no further discussion.

That leaves us with two possibilities - either questions are reducible to theories, or they are a distinct epistemic element irreducible to theories. As presently defined, theory is a set of propositions (Sebastien, 2016). So it follows that any attempt to reduce questions to theories will necessarily be an attempt to reduce questions to propositions. The question of whether questions/topics can be accommodated within a propositional framework is the primary animating questions of this essay. If it turns out to be the case that questions can be reduced to theories then the basic ontology of theories and methods as we accept it nowadays will hold, while questions will turn out to be a subclass of theories. If, however, it is discovered that questions cannot be expressed propositionally then the scientonomic ontology of epistemic elements will need to be revised to accommodate questions.

\section{Two Hypotheses}

Given that we have already settled that questions must both be accommodated into the mosaic and cannot be reduced to methods, we are left with the question of whether questions can be reduced to theories. Before we begin, we should have a basic understanding of what such a reduction would look like and what requirements it must meet. Intuitively, our epistemic elements should be exhaustive. If an element is relevant to the mosaics of epistemic communities - and it certainly seems that questions are relevant - then theoretical scientonomists should have a means to describe it. If even a single relevant element escapes our descriptive framework then it follows that the framework is incomplete. If questions were reducible to theories, it would have to be demonstrated that propositionally questions are not distinct from theories. That is to say, it would have to be shown in the general form that all questions have identical semantic content when restated as propositions. Following Groenendijk and Stokof (1997), I will call this the reductionist thesis.

In the literature on questions, two possible avenues for the reductionist have been proposed: the reductionist can treat questions either as descriptive propositions that describe an absence of knowledge on a certain subject on the part of the knower and a desire to gain said knowledge (Künne, 2003) or as normative imperatives for a 
third party to disclose some piece of knowledge (Åqvist, 1965; Åqvist, 1971; Hintikka \& Halonen, 1995). I will follow the convention described by Cross and Roelofsen (2016) in calling the first treatment the descriptiveepistemic account and the second the imperative-epistemic account.

On the descriptive-epistemic account we can take some question generally accepted as legitimate and we can consider that question as a proposition describing the state of the scientific community's knowledge on that subject. Consider the problem of $\mathrm{CP}$ violation:

(1) Why are there no observed instances of CP violation in QCD?

According to the descriptive-epistemic approach, we can analytically decompose (1) such that the content of the question is expressed through a conjunction of descriptive propositions. To do so, the reductionist must reformulate the question in such a way, as to meet two conditions:

- preservation-of-semantic-content condition: the reformulation preserves the semantic content of the question in such a way that it is true or false;

- preservation-of-the-interrogative condition: the reformulation preserves the interrogative nature of the question.

If the descriptive-epistemic reductionist wants to succeed in expressing the question propositionally then they should satisfy both of these conditions by interpreting the question epistemically, i.e. by interpreting it as being about the state of knowledge of a certain epistemic agent - in this case, the contemporary physics community. To do so, the descriptive-epistemic reductionist may try to express (1) as a conjunction of the following descriptive propositions:

(2a) The contemporary physics community does not have an accepted answer as to why there are no observed instances of CP-violation.

and

(2b) The contemporary physics community seeks to find an acceptable answer as to why there are no observed instances of CP-violation

The descriptive-epistemic reductionist claims that the conjunction (2a) and (2b) satisfy both conditions. Specifically, (2a) is said to satisfy the preservation-of-semantic-content condition and (2b) is said to satisfy the preservation-of-the-interrogative conditions. In both cases the major step in interpreting the semantics involved is shifting the content from the elements of the proposition to the status of the epistemic agent. (2a) will be true just in case the epistemic agent putting forward the proposition is in fact ignorant as to why there are no observed instances of CP violation in QCD. Similarly, (2b) will be true just in case the epistemic agent putting forward the assertion is seeking an answer to that question.

Alternatively, the reductionist may adopt the imperative-epistemic approach and to treat the interrogative as an imperative proposition demanding an answer. To accomplish this, the reductionist may seek to reformulate (1) in either of the following ways:

(3a) Let it be the case that the contemporary physics community has an acceptable explanation for why there are no observed instances of $\mathrm{CP}$ violation in QCD!

or

(3b) The contemporary physics community ought to find an acceptable answer as to why there are no observed instances of CP-violation.

Either of (3a) or (3b) might be viewed by the imperative-epistemic reductionist as an acceptable way of

Åqvist, in his (1965), attempts to put forward a complete formalized system of interrogative logic that is based on the imperative-epistemic account. formulating a question as an imperative. This is because, in both cases, the essential characteristics of the interrogative - the implication that there is some 
knowledge that is unknown to the asker and exhortation to disclose some piece of knowledge - is meant to be preserved.

In the following two sections, I will argue that neither of these approaches succeeds in reducing questions to propositions. First, these alleged reductions fail to preserve the semantic content of questions and instead replace that content with historical descriptions or imperatives. Second, by portraying questions as propositions, the reductionist approach ends up in an infinite regress where each question is also an answer to another question, which is an answer to yet another question, ad infinitum.

\section{Question vs. Historical Record}

Let us first appreciate that both the descriptive-epistemic and imperative-epistemic attempts to reduce questions to propositions fail to maintain the actual semantic content of questions, but rather replace it with either a description of the agent's mosaic, or an imperative prescribing that the agent ought to pursue a certain direction of research. In both the descriptive-epistemic and the imperative-epistemic accounts, the major interpretive step is to shift the semantic content from the question's object (i.e. the fact that there are no observed instances of CP violation in $\mathrm{QCD}$ ) to the mosaic of the epistemic agent asking the question (i.e. the mosaic of the contemporary physics community). What both of these accounts ignore is the fact that the semantic content of a question is not the same as its contingent historical fate.

After all, the same question can be accepted as legitimate by many different epistemic agents at very different times and places. Such questions as "why do rocks fall to the ground?" or "why do planets change their apparent positions?" have been accepted as legitimate topics of research by many different communities at different timeperiods. Thus, it is safe to say that the semantic content of a question is not to be confused with the historical record of its acceptances by different epistemic agents at different times. The following UML diagram shows the relation between the concept of question and the concept of acceptance record:

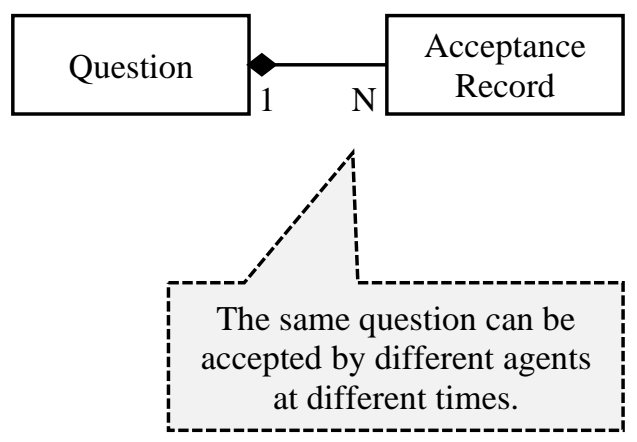

What both the descriptive-epistemic and imperative-epistemic accounts fail to recognize is that the semantic content of the question stays the same regardless of its historical fortunes. The alleged reductions of (1) fail to capture this simple point. They don't offer any meaningful way of describing the content of a question without referring to the specific historical context of a certain epistemic agent. Thus, (2a) and (2b) describe the state of the mosaic of the contemporary physics community. To argue that the semantic content of (1) has been fully captured in (2a) and (2b) is to admit that one has no way of speaking of this same question as being potentially accepted by any community other than the contemporary physics community. In this reductionist picture, each question becomes a description of the respective agent's mosaic at a specific moment of time. As a result, according to the reductionists, there is no meaningful way of saying "question $q$ has been accepted by epistemic agents $x, y$, and $z$ ". Nor do they offer any meaningful way of saying "question $q$ has been accepted by epistemic agent $x$ both during 1700-1740 and then again after 1920". This absurd outcome stems from a failure to appreciate that the semantic content of a question is not the same as the historical record of the question's acceptances by different epistemic agents at different periods of time.

Consider the conjunction of (2a) and (2b) - both are descriptive historical propositions describing the absence of an accepted explanation for the lack of observed instances of CP-violation. In other words, the propositions 
deal with the contents of the mosaic; specifically, whether a theory of a certain sort can be found in the mosaic. The truth-conditions associated with the individual propositions (and their conjunction) depend on whether the mosaic has the element named in the proposition; in this case, a theory explaining why there are no observed instances of CP-violation. If the contemporary physics community had an accepted theory explaining our failure to observe any instances of CP violation in QCD as part of its mosaic, then the propositions given by (2a) and (2b) would be false. Likewise, if the contemporary physics community has no accepted theory explaining the same as part of its mosaic but is also not pursuing any theory then (2a) would be true but (2b) would be false, and so the conjunction of the two would be false as well.

We might also consider the alleged imperative-epistemic reductions ( $3 a)$ and $(3 b)$. While the truth conditions of these cannot be cashed out as straightforwardly as the truth conditions of the conjunction of (2a) and (2b), we can see that again the truth conditions are relative to the inclusion or exclusion of certain theories from the mosaic. Following the imperative semantics set out by Åvist in his (1965) and (1971), we can say that the statements with imperative operator such as (3a) have truth value relative to a particular context. If the statement is satisfiable (can be brought about) in every context under consideration, then the statement is true. The context under consideration in (3a) is the context of a mosaic - the epistemic nature of the interpretation of the interrogative necessarily entails that it be interpreted this way. Put another way, (3a) would not be satisfiable, and therefore false, if there were a context in which the imperative could not be satisfied. Since the imperative specifies that the contemporary physics community find the explanation acceptable, we've already specified a context of satisfaction in which satisfiability will depend on the inclusion or exclusion of a theory from the mosaic. Likewise, (3b) would be false if the negation was true; if the contemporary physics community ought not to find a solution to the problem then the proposition will be false, and again the question becomes about whether a theory should or should not be pursued for possible inclusion in the mosaic.

The problem for the reductionist is that, in their bid to reduce questions to propositions, they fail to preserve the semantic content, the meaning of the question. Regarding the original question (1) we have kept under consideration - that of why there have been no observed instances of CP-violation - we need to consider what the question means, or rather what it does not mean. The way it is stated in (1), it does not contain any reference to any specific mosaics, but merely expresses a potential topic of research. This is the semantic content of the question that needs to be preserved; it should not be confused with propositions describing the historical fate of this question, i.e. which community and when accepted the question as legitimate.

Of course, the reductionist may well contend that shifting the semantic content of a question by reducing it to historical propositions is an unsavoury but nevertheless necessary step. It does not mesh well with our intuitions about what questions mean, but that does not make the reductionist approach outright untenable. We may, after all, simply have misguided intuitions about the semantic content of questions, in particular as they relate to science. The reductionist could contend that as unpalatable as it is to say that asking questions only ever reflects historical descriptions and imperatives, it would be more unpalatable to fundamentally alter the ontology of our epistemic elements. The argument discussed in the next section shows why the reductionist approach is untenable, rather than just unattractive.

\section{The Infinite Regress}

Let us first consider the semantic structure of question-answer exchanges from erotetic logic (logic of questions). To do this we will focus on a special kind of scientific question - the why-question (WQ). According to

According to Bromberger, the form of the WQ-structure can be given as an ordered pair $\langle q, e>$ where $q$ is the question and $e$ is the explanation. Bromberger also specified a third element $a$, suggesting an ordered triple $<a, q, e>$ with $a$ as the true presuppositions of the question (1966, p. 87). For our purposes the extra element is extraneous. Bromberger also attempted to elucidate the structure of the set $e$, but that too is extraneous for our purposes.
Bromberger, the typical form of the WQ can have any number of potential answers - correct or incorrect, accepted or unaccepted, pursued or neglected. Thus, the answer to the question "why is it the case that q?" can be of the form "Because e" where $q$ is the phenomenon to be explained and $e$ is "a proposition reserved for declarative sentences" (Bromberger, 1966, p. 87). We can call these www.scientojournal.com • Status of Questions 
together the WQ-structure. Based on this, we can expect that the latter half of the WQ-structure corresponding to the question posed by (1) - i.e. its answer - will have the form "there are no observed instances of CP violation in QCD because e" where $e$ is the explanation, as given by a set of propositions. This fits nicely with the current scientonomic definition of theory as "a set of propositions" (Sebastien, 2016).

Obviously WQs are not the only kind of question we can consider given that who, what, what is, where, when, whether, and a slew of other types of questions are also abundant in the practice of science. How-questions, one can argue, are very much like WQs especially when asked

Bromberger excludes from this formalism any why-question whose answer is motivational or nonsensical (1966, p. 87). This excludes rhetorical questions which are not intended to have an answer, like when a teacher asks an unruly pupil "why must you insist on causing such a disruption?".

in a scientific context. For example, the question of "how do massive objects distort space-time?" and "why do massive objects distort space-time?" both seek essentially the same answer. The use of 'how' specifically might imply that the asker is looking for a more mechanical answer, but this is not an especially relevant detail. WQs will be the focus of this section.

My focus on why-questions is not arbitrary; WQs have been famously characterized as that sort of question which precedes scientific explanations (Hempel, 1989). In fact, according to Hempel's definition, being the answer to a WQ is what characterizes a scientific explanation. Though that characterization has been criticized in the literature it nevertheless serves as a good starting point that captures our intuitive sense of what it means to explain something (Woodward, 2017). Further, Bromberger has taken great strides towards developing a fully formalized theory about how WQs are answered and the sorts of propositions that rightly qualify as answers to scientific why-questions (Bromberger, 1966). Although Bromberger's theory is not without its acknowledged limitations (Teller, 1974) it is useful in its construction of the WQ-structure. In any event, since WQs are abundant in all sciences, what we learn about WQs will tell us a lot about questions in general.

Now, if the reductionist project is to succeed in showing that questions are reducible to propositions, then they should be able to fit any reduced why-question into this WQ structure of question and answer. However, since the reductionist thesis is that questions are fully reducible to propositions, there should be no functional difference between questions and their answers; the reductionist simply conceives of the set of questions as being a subset of propositions. This presents a challenge for the reductionist: how are they to accommodate the allegedly reduced, propositional forms of questions into the WQ-structure?

Let's consider our main example - question (1). The descriptive-epistemic reductionist could try to construct a WQ-structure using the conjunction of (2a) and (2b) as standing for the question together with some potential answer (4):

(2a) The contemporary physics community does not have an accepted answer as to why there are no observed instances of CP-violation in QCD.

(2b) The contemporary physics community seeks to find an acceptable answer as to why there are no observed instances of CP-violation in QCD.

(4) There are no observed instances of CP violation in QCD because $e$.

Two problems emerge from this structure. First, it is not immediately clear is how (4) could possibly provide an answer to the conjunction of (2a) and (2b). Since all of these are propositions in the classical sense, their truth conditions will be bound up with whether they obtain, but there is no implication that connects (4) with (2a) and (2b). The reductionist could claim that (2b) presents a dispositional attitude towards seeking out (4), but this is a weak defense at best. We can repeat this process for the imperative-epistemic reductions with similar effect:

(3a) Let it be the case that the contemporary physics community has an acceptable explanation for why there are no observed instances of $\mathrm{CP}$ violation in QCD!

(4) There are no observed instances of CP violation in QCD because $e$.

In this case it is more plausible that (3a) reflects a dispositional attitude towards (4), but then we run into a problem of what the truth-conditions for (3a) would be. If the semantic content of (3a) is given by a set of truth 
conditions, as has been assumed throughout this paper, then it's unclear how (4) and (3a) can be logically linked. If, on the other hand, the semantic content of (3a) is given by a dispositional attitude then it is not a proposition at all and is therefore outside the scope of the argument. We can then consider the second imperative-epistemic possibility:

(3b) The contemporary physics community ought to find an acceptable answer as to why there are no observed instances of CP-violation in QCD.

(4) There are no observed instances of CP violation in QCD because $e$.

If the reductionist were to put forward this version of the WQ-structure, then they would have a structure which has clear truth-conditional semantics and a dispositional attitude towards obtaining an answer. However, in this case they would fall victim to a version of the familiar Humean maxim that one cannot derive an ought from an is, or vice versa. Still, it is worth pondering over just how the descriptive proposition (4) is meant to provide an answer to the normative proposition (3b).

The second and arguably more daunting problem for the reductionist is that if we look at how the questions and answers are structured, then the reductionist view forces us to treat any proposition as an answer to a question which, as a proposition itself, can be construed as the answer to another independent question. This implies that in the particular WQ-structure if the element $q$ is the question in one structure, it is also the answer, $e$, in another structure, which has its own question to which $q$ is the answer. This follows from the most common definition of a proposition as non-linguistic bearers of truth value (McGrath, 2014), which means that any proposition is, if nothing else, an answer to a question in the form of " $p$, or not $p$ ?" In other words, every proposition at very least answers the question of whether that proposition is true. For example, if the reductionist were to apply the descriptive-epistemic interpretation, they could construe the descriptive proposition (2a) to be an answer to the question "do we know why there are no observed instances of $C P$-violation in $Q C D$, or not?" Likewise, if they reduce this latter question to propositions we end up with the conjunction of "we do not know whether we know why there are no instances of CP violation in QCD or not" and "we want to know whether we know why there are no observed instances of CP violation in QCD" and so on.

This applies to questions interpreted on the imperative-epistemic account as well. If we consider the alleged reduction (3b), it can be construed as an answer to the question "ought it to be brought about that we know why there are no observed instances of $C P$ violation in $Q C D$ ?" The reductionist would consider this to be identical to the proposition "it ought to be brought about that we know whether it ought to be brought about that we know why there are no observed instances of CP violation in QCD." This could then be construed as the answer to another question, itself analytically reducible to another proposition, and so on.

Propositions of the form ! $\Phi$, like (3a), will fall into this problem in different ways depending on how one construes an imperative to follow from another imperative. Different systems of imperative logic will yield different rules of inference, but which one we pick and how the rules of inference work is not relevant here.
The result of this continuous process of alleged reductions is that the reductionist ends up constructing an infinite regress of nested epistemic questions and answers. The result is the absurd situation where any question must have an antecedent question to which it is an answer, and so on. This applies to any attempt to reduce questions to propositions. If we pick any proposition at all, it is an answer to a question, which - if we were to believe reductionists - is itself a proposition and, thus, an answer to another question, which is itself a proposition and, thus, an answer to a third question, ad infinitum. The reductionist thesis forces us to consider any question as implying this infinite backwards regress. The only way to escape the regress it to finally admit that the reductionist project is doomed. Therefore, the only reasonable conclusion is that questions are not proposition, and so they cannot possibly be interpreted as a subclass of theories.

\section{Conclusion}

The question of the status of questions can only be resolved by admitting questions into the scientonomic ontology of epistemic elements as a distinct class in par with theories and methods. This step will allow us to avoid the 
unacceptable consequences of the reductionist position, primarily the problem of infinite regress as well as the problem of confusing the semantic content of a question with its historical record. It will also help us reconcile our theoretical ontology with the ontology implicit in our encyclopedia. Here is the new ontology of epistemic elements that I suggest:

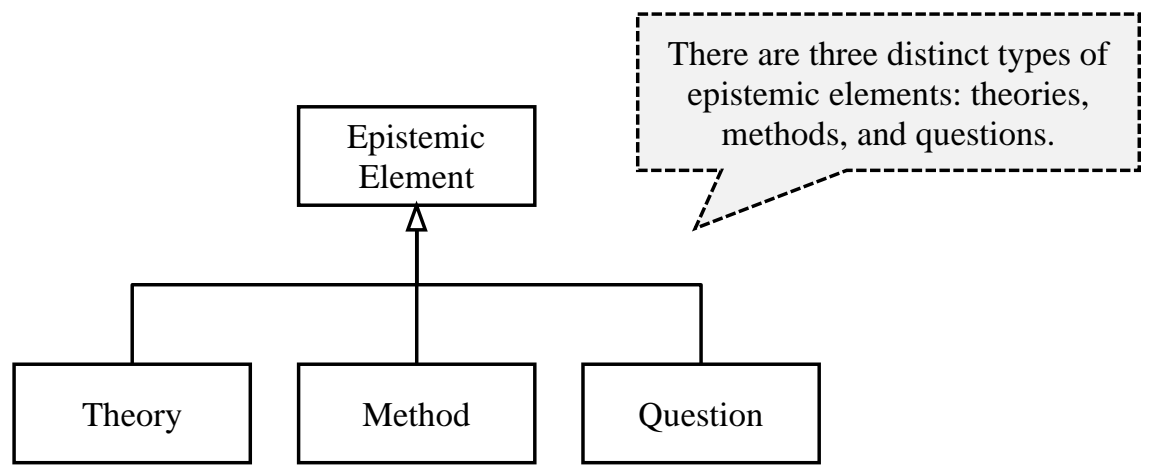

In this paper, it has not been my goal to show how communities come to accept questions as legitimate. If my suggested modification becomes accepted, the question of the mechanism of question acceptance/legitimacy will itself become legitimate and will demand no small amount of effort on the part of our community to resolve. For now, it will have to remain mute on how exactly the acceptance of questions take place and how it relates to the employment of methods or the acceptance of theories.

Here, I will tentatively propose that the only possible stance that epistemic agents can take towards questions is question acceptance, defined as follows:

\begin{tabular}{|c|}
\hline Question Acceptance $\equiv$ \\
\hline A question is said to be \\
accepted if it is taken as a \\
legitimate topic of inquiry. \\
\hline
\end{tabular}

The opposite of this stance is unacceptance: a question is said to be unaccepted when it is not considered a legitimate topic of inquiry. This will help us avoid a possible confusion with theory acceptance/unacceptance. Once again, these are broad stances and their binary nature may be limiting, but they reflect the actual practice of our community with respect to how we treat questions (consider that topics in the encyclopedia are regarded as legitimate or illegitimate).

At this time, the laws and theorems of scientonomy concern theories and methods only and say nothing whatsoever about questions. Admitting questions into the ontology of epistemic elements would mean entering uncharted territory; there is clearly a possibility for new laws and theorems that will explain how questions become legitimate/illegitimate and how this process affects and is affected by theory acceptance and method employment. There is a great potential for extending the scope of our theory and shedding new light on the process of scientific change. Among other things, it would mean admitting that theoretical scientonomy may play a larger role in understanding theory pursuit than was previously thought. It would also mean opening a whole new layer of potential discoveries for observational scientonomy, such as learning different historical indicators of when a question is considered legitimate and what the associated markers for question legitimacy are. But before we get there, we must first accept questions into the scientonomic ontology of epistemic elements as a distinct element.

\section{Acknowledgements}

I am greatly indebted to Hakob Barseghyan for his many helpful comments and suggestions, as well as the review committee for their critiques of earlier versions of this paper. I am also greatly indebted to the blind reviewer who provided several helpful comments. 


\section{Suggested Modifications}

Therefore, I suggest the following modifications:

\section{[Sciento-2018-0001]}

Accept the following definition of question:

- Question $\equiv$ a topic of inquiry.

\section{Question $\equiv$}

A topic of inquiry.

\section{[Sciento-2018-0002]}

Accept the following ontology of epistemic elements with theories, methods, and questions as distinct epistemic elements:

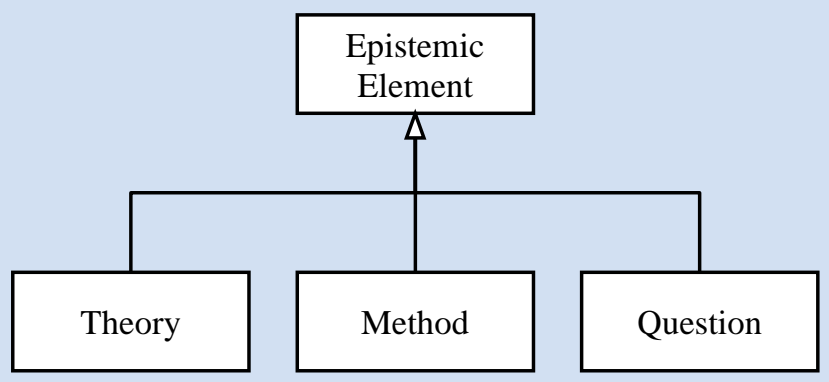

Reject the previously accepted ontology of epistemic elements.

\section{[Sciento-2018-0003]}

Provided that modification [Sciento-2018-0002] is accepted, accept that the epistemic stance that can be taken by an epistemic agent towards a question is question acceptance (the opposite is unacceptance), defined as follows:

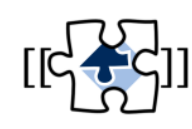

- Question Acceptance $\equiv$ a question is said to be accepted if it is taken as a legitimate topic of inquiry.

\begin{tabular}{|c|}
\hline Question Acceptance $\equiv$ \\
\hline A question is said to be \\
accepted if it is taken as a \\
legitimate topic of inquiry. \\
\hline
\end{tabular}

\section{[Sciento-2018-0004]}

Provided that modifications [Sciento-2018-0002] and [Sciento-2018-0003] are accepted, accept the following question as legitimate topics of scientonomic inquiry:

- Mechanism of Question Acceptance: How do questions become accepted as legitimate? What is the mechanism of question acceptance?

- Indicators of Question Acceptance: What are the historical indicators of theory acceptance? How can observational scientonomists establish that such-and-such a question was accepted as a legitimate topic of inquiry by a certain epistemic agent at a certain time? 


\section{References}

Åqvist, L. (1965). A New Approach to the Logical Theory of Interrogatives. Part I: Analysis. Philosophical Studies, University of Uppsala.

Åqvist, L. (1971). Revised Foundations for Imperative-Epistemic and Interrogative Logic. Theoria 1, pp. 33-73.

Barseghyan, H. (2015). The Laws of Scientific Change. Springer.

Bromberger, S. (1966). Why-Questions. In Colodny (Ed.) (1966), pp. 86-111.

Colodny, R. (Ed.) (1966). Mind and Cosmos: Essays in Contemporary Science and Philosophy. University of Pittsburg Press.

Cross, C. \& Roelofsen, F. (2016). Questions. In Zalta, E. N. (Ed.) (2016). The Stanford Encyclopedia of Philosophy (Spring 2016 Edition). Retrieved from https://plato.stanford.edu/archives/spr2016/entries/questions/.

Groenendijk, J. \& Stokhof, M. (1997). Questions. In van Benthem \& ter Meulen (Eds.) (1997), pp. 1055-1124.

Hempel, C. G. (1989). Aspects of Scientific Explanation. In Kitcher \& Salmon (Eds.) (1989), pp. 331-489.

Hintikka, J. \& Halonen, I. (1995). Semantics and Pragmatics for Why-Questions. The Journal of Philosophy 92(12), pp. 636-657.

Kim, J. E. \& Carosi, G. (2010). Axions and the Strong CP Problem. Reviews of Modern Physics 82, pp. 557-596.

Kitcher, P. \& Salmon, W. (Eds.) (1989). Scientific Explanation. University of Minnesota Press.

Kuhn, T. (1970). The Structure of Scientific Revolutions. Second Edition. The University of Chicago Press.

Künne, W. (2003). Are Questions Propositions? Revue Internationale de Philosophie 224, pp. 157-168.

McGrath, M. (2014). Propositions. In Zalta, E. N. (Ed.) (2014). The Stanford Encyclopedia of Philosophy (Spring 2014 Edition). Retrieved from https://plato.stanford.edu/archives/spr2014/entries/propositions/.

Patton, P.; Overgaard, N.; \& Barseghyan, H. (2017). Reformulating the Second Law. Scientonomy 1, pp. 29-39. Retrieved from https://www.scientojournal.com/index.php/scientonomy/article/view/27158.

Sebastien, Z. (2016). The Status of Normative Propositionsin the Theory of Scientific Change. Scientonomy 1, pp. 1-9. Retrieved from https://www.scientojournal.com/index.php/scientonomy/article/view/26947.

Teller, P. (1974). On Why-Questions. Nous 8, pp. 371-380.

van Benthem J. \& ter Meulen A. (Eds.) (1997). The Handbook of Logic and Language. MIT Press.

Woodward, J. (2017). Scientific Explanation. In Zalta, E. N. (Ed.) (2017). The Stanford Encyclopedia of Philosophy (Spring 2017 Edition). Retrieved from https://plato.stanford.edu/archives/spr2017/entries/scientific-explanation/. 